\title{
Can a Lifestyle Genomics Intervention Motivate Patients to Engage in Greater Physical Activity than a Population-Based Intervention? Results from the NOW Randomized Controlled Trial
}

\author{
Justine R. Horne ${ }^{\mathrm{a}-\mathrm{c}}$ Jason Gilliland ${ }^{\mathrm{c}-\mathrm{i}}$ Tara Leckie $^{\mathrm{j}} \quad$ Colleen O'Connor $\mathrm{O}^{\mathrm{c}, \mathrm{g}, \mathrm{j}}$ \\ Jamie A. Seabrook ${ }^{c, d, g-j}$ Janet Madillc, $g, j$ \\ ${ }^{a}$ Health and Rehabilitation Sciences, The University of Western Ontario, London, ON, Canada; ${ }^{\mathrm{b}}$ The East Elgin \\ Family Health Team, Aylmer, ON, Canada; ${ }^{C}$ Human Environments Analysis Laboratory, The University of Western \\ Ontario, London, ON, Canada; ${ }^{\mathrm{d}}$ Department of Paediatrics, The University of Western Ontario, London, ON, Canada; \\ e School of Health Studies, The University of Western Ontario, London, ON, Canada; ${ }^{f}$ Department of Geography, \\ The University of Western Ontario, London, ON, Canada; ${ }^{9}$ Lawson Health Research Institute, London, ON, Canada; \\ ${ }^{h}$ Children's Health Research Institute, London, ON, Canada; 'Department of Epidemiology and Biostatistics, The \\ University of Western Ontario, London, ON, Canada; 'School of Food and Nutritional Sciences, Brescia University \\ College at The University of Western Ontario, London, ON, Canada
}

\section{Keywords}

Physical activity · Lifestyle genomics · Obesity · Randomized clinical trial · Genetics

\section{Abstract \\ Background: Lifestyle genomics (LGx) is a science that ex- plores interactions between genetic variation, lifestyle com- ponents such as physical activity (PA), and subsequent health-and performance-related outcomes. The objective of this study was to determine whether an LGx intervention could motivate enhanced engagement in PA to a greater ex- tent than a population-based intervention. Methods: In this pragmatic randomized controlled trial, participants received either the standard, population-based Group Lifestyle Balan- $\mathrm{ce}^{\mathrm{TM}}$ (GLB) program intervention or the GLB program in ad- dition to the provision of $L G x$ information and advice (GLB + LGx). Participants ( $n=140)$ completed a 7-day PA recall at baseline, 3, 6, and 12 months. Data from the PA recalls were used to calculate metabolic equivalents (METs), a measure of energy expenditure. Statistical analyses included split plot analyses of covariance and binary logistic regression (gener-}

karger@karger.com www.karger.com/lfg

Karger ${ }^{\prime \prime} \div$

BOPEN ACCESS
(C) 2020 The Author(s)

Published by S. Karger AG, Basel

This article is licensed under the Creative Commons AttributionNonCommercial-NoDerivatives 4.0 International License (CC BYNC-ND) (http://www.karger.com/Services/OpenAccessLicense) Usage and distribution for commercial purposes as well as any distribution of modified material requires written permission. alized linear models). Differences in leisure time PA weekly METs, weekly minutes of moderate + high-intensity PA, and adherence to $P A$ guidelines were compared between groups (GLB and GLB + LGx) across the 4 time points. Results: Weekly METs were significantly higher in the GLB + LGx group $(1,114.7 \pm 141.9 ; 95 \% \mathrm{Cl} 831.5-1,397.8)$ compared to the standard GLB group (621.6 $\pm 141.9 \mathrm{MET} /$ week; $95 \% \mathrm{Cl} 338.4-$ $904.8)$ at the 6 -month follow-up $(p=0.01)$. All other results were non-significant. Conclusions: The provision of an LGx intervention resulted in a greater weekly leisure time PA energy expenditure after the 6-month follow-up. Future research should determine how this could be sustained over the long-term. Clinical Trial Registration: NCT03015012.

(c) 2020 The Author(s)

Published by S. Karger AG, Basel

\section{Introduction}

Regular physical activity (PA) is associated with numerous health benefits, including reduced stress and increased energy levels, as well as a reduced risk of cardiovascular disease, arthritis, and obesity [1]. Physical inac- 
tivity is a major modifiable risk factor for several chronic diseases such as obesity, hypertension, cardiovascular disease, and type 2 diabetes [1]. Although the health benefits of PA and increased energy expenditure are well established, nearly $30 \%$ of the population globally is not achieving the recommended PA guidelines [2]. Concerns related to physical inactivity are greatest in high-income Western countries, with over $40 \%$ of the population failing to achieve the recommended PA guidelines, and substantial reductions in PA energy expenditure observed over recent decades [2]. As such, novel, innovative strategies are needed to motivate the general public to participate in PA in order to improve the health and well-being of the population.

There have been considerable scientific advancements exploring how genetics and PA interact to impact health and performance outcomes. It is well established that genetic variation within the fat mass and obesity-associated (FTO) gene is associated with a significantly increased risk of obesity [3]. Furthermore, single-nucleotide polymorphisms located within certain genes can impact individual responses to different PA approaches for weight management [4-7]. For example, research has consistently demonstrated that increased PA can attenuate the obesity-associated effects in high-risk FTO genotypes and that PA can enhance weight loss in individuals with specific single-nucleotide polymorphisms within the FTO gene [4-11]. Genetic variation can also help us to understand individual variations in athletic predisposition. Single-nucleotide polymorphisms located within the genes adrenergic receptor beta 3 (ADRB; rs4994), nuclear factor erythroid 2-related factor 2 (NRF2; rs12594956), glutathione S-transferase pi 1 (GSTP1; rs1695), and nuclear factor I A antisense RNA 2 (NFIA-AS2; rs1572312) have been associated with genetic predisposition to excel at aerobic/ endurance-based activities [12-15]. When it comes to speed and power phenotypes, the CC and TC genotypes in the $\alpha$-actinin-3 (ACTN3; rs 1815739) gene express an association with genetic predisposition to excel at activities requiring strength and speed [15-19]. This science, referred to as lifestyle genomics (LGx), is the study of interactions between genetic variations, lifestyle factors, and health and performance outcomes [20]. The abovementioned LGx information and advice are available to the public through consumer genetic testing.

Genetic testing holds promise as a lifestyle intervention with the continual advancement of genomic science research. A recent systematic review found that providing personalized lifestyle recommendations based on genetics can help to encourage lifestyle behavior changes, espe- cially when the genetic information includes actionable lifestyle recommendations [21]. Genetic research assessing behavioral changes should also remain cognizant of established behavior change theory in order to provide participants with interventions that are likely to facilitate health behavior changes $[21,22]$. With this in mind, the purpose of the present study, a secondary outcome analysis of the Nutrigenomics, Overweight/Obesity and Weight Management (NOW) trial, was to determine whether providing actionable, genetic-based PA information and advice motivates primary care patients to engage in greater PA compared to the provision of population-based information and advice. The interventions included in this trial were designed to optimize health behavior changes based on the Theory of Planned Behavior (TPB).

\section{Methods}

\section{Study Design}

The NOW trial was a pragmatic randomized controlled trial involving 140 participants with follow-up after 3, 6, and 12 months. Participants were primarily middle-aged, middle-income, Caucasian women with obesity (class II). The full study details, including a CONSORT flow diagram, sample size calculation based on the primary outcome of the trial (body composition, $n=74$ ), and a summary of participant characteristics, have been published elsewhere [23-25]. In brief, the sample consisted of participants who were recruited from the Group Lifestyle Balance ${ }^{\mathrm{TM}}(\mathrm{GLB})$ program at the East Elgin Family Health Team in Aylmer, ON, Canada. The GLB program was 12 months in duration and aimed to provide lifestyle information and advice for weight management through 23 group-based sessions. Through computer-generated block randomization (using randomly permuted blocks) conducted by the first author, half of the GLB program groups were randomized (1:1) to receiving population-based lifestyle information and advice (GLB group) and the other half were randomized to receiving genetic-based lifestyle information and advice (GLB + LGx group). Four of the authors (J.G., J.A.S., C.O., and J.M.) were blinded to the group assignments. Patients expressing interest in the GLB program were invited to participate in this study by the first author if they met the following inclusion criteria: $\mathrm{BMI} \geq 25.0$, age $\geq 18$ years, English-speaking, willing to undergo genetic testing, having access to the internet, and not seeing another healthcare provider for weight loss advice outside of this study. Pregnancy and lactation were exclusion criteria. Recruitment ended after enough participants had been recruited for 10 randomized GLB groups, at which point the sample size had been exceeded. PA recommendations were communicated during GLB group sessions and reinforced during 3 follow-up one-on-one sessions at 3, 6, and 12 months. The interventions for the standard GLB and GLB + LGx groups were identical in duration $(1 \times 90$-min and $22 \times 60$-min group-based sessions and $4 \times 30$-min one-on-one sessions). Staggered cohorts were implemented between May 2017 and September 2019, while balancing seasonality between the 2 intervention groups. This study is registered with clinicaltrials.gov. (NCT03015012). 


\section{Population-Based Intervention (Standard GLB Group)}

Standard, population-based lifestyle advice was provided to participants in the standard GLB group intervention by the first author, who was a certified GLB program facilitator. The population-based reports were reviewed at the 3 follow-up one-on-one appointments occurring after 3, 6, and 12 months. With respect to $\mathrm{PA}$, participants were advised to aim to complete at least $150 \mathrm{~min} /$ week of moderate-intensity PA with resistance activities at least 2 days a week. They were further advised to find endurance and strength-based activities that they enjoy in order to meet this guideline. The following 3 components of the TPB were incorporated into the intervention strategy: subjective norms, perceived behavioral control, and attitudes [26]. To affect change in subjective norms, the intervention was primarily group-based and allowed for a supportive group environment. Participants shared and discussed their progress with their peers as they worked toward similar goals and behaviors. To affect change in perceived behavioral control, the group sessions focused on gradually increasing PA levels that were safe and realistic. Lastly, to affect change in attitudes towards PA, participants were informed of the positive health impacts of PA and its importance for weight management. The complete population-based guidelines that participants received have been published elsewhere [23].

\section{$L G x$ Intervention (GLB + LGx Group)}

Participants randomized to the GLB + LGx group intervention received personalized genetic-based PA information and advice, with the report reviewed at the 3 follow-up one-on-one appointments occurring after 3, 6, and 12 months. Participants were counselled on actionable genetic-based recommendations, which were based on a genetic testing report that is commercially available to the general public through healthcare professionals. Participants with the AA genotype in the FTO gene ( $r s 9939609$ ) were instructed to achieve a PA target of 30-60 min/day, 6 days a week (180-360 $\mathrm{min} /$ week) of moderate-intensity activity, with strengthening activities at least 2 days a week. Participants were informed that achieving this higher PA target (compared to population-based recommendations) could lead to an "enhanced weight loss response." Participants with the TA or TT genotype of FTO (rs9939609) were instructed to work toward the abovementioned population-based PA guidelines (150 min weekly with musclestrengthening activities 2 days a week). The genetic intervention also aimed to positively impact key components of the TPB including: subjective norms, perceived behavioral control, and attitudes toward PA [26]. In addition to the effects on subjective norms, the perceived behavioral control and attitudes mentioned in Population-Based Intervention (Standard GLB Group), the genetic intervention further aimed to affect these components through the provision of individualized DNA-based information and recommendations for PA. To optimize changes in perceived behavioral control and attitudes, individuals in the genetic group were also informed about whether or not they had a genetic predisposition to excel at endurance and/or strength-based activities. The genetic variants that were included in the genetic report and used to assess genetic predisposition to excel in endurance/aerobic-based activities were: ADRB (rs4994), NRF2 (rs12594956), GSTP1 (rs1695), and NFIA-AS2 (rs1572312). Furthermore, participants were provided with information on their genetic predisposition to excel at strength and power-based PA based on ACTN3 (rs1815739) genetic variation. For example, individuals with the TC or CC variant of ACTN3 (rs1815739) were informed: "You have an enhanced genetic predisposition to excel in strength and power-based PA." Those who did not have such genetic variants were informed that they had a typical genetic predisposition to excel in strength/power or endurance activities. A sample genetic report that participants received has been published elsewhere [23]. The standard GLB program was modified to replace standard population-based advice.

\section{Genotyping}

Oragene ON-500 saliva collection kits (DNA Genotek, Ottawa, ON, Canada) were used to collect DNA samples of the participants at the East Elgin Family Health Team. The saliva samples were shipped to the University of Toronto and stored at $-80^{\circ} \mathrm{C}$. The iPLEX Gold assay with mass spectrometry-based detection on the Sequenom MassARRAY ${ }^{\circledR}$ platform was used for genotyping of the single-nucleotide polymorphisms listed above.

\section{Data Collection and Outcomes}

Participants were followed up for 12 months, with data collection occurring at baseline (during a 2 -week run-in period in which group allocation was concealed for the participants) and 3, 6, and 12 months. Staggered cohorts were implemented between May 2017 and September 2019. Data were collected through self-reported past-week PA recalls of leisure-time activity only. This included the type of activity, duration, and intensity (light, moderate, or vigorous). Leisure time PA was defined as any planned activity completed outside of work. In cases where participants did not follow the instructions to include only leisure time PA and also listed job-related activity, the job-related activity was excluded from the analyses, as further detailed below, given that evidence suggests that recreational (leisure time) PA has greater health benefits than non-recreational PA [27]. Change in metabolic equivalents (METs) between groups was a predetermined secondary outcome of the NOW trial; changes in minutes of leisure time PA and PA adherence were exploratory analyses.

\section{Data Analysis}

All analyses were by originally assigned groups. The Compendium of Physical Activities [28] was used to calculate METs (a measure of energy expenditure) of participants' leisure time PA. One MET is defined as the amount of oxygen used by the body while sitting at rest and it is equal on average to $3.5 \mathrm{~mL}$ oxygen $/ \mathrm{kg} /$ min [28]. Activities on the compendium were matched to the participant's type and intensity of activity to find an appropriate MET value. Following this, the duration in minutes of the participants' activity was multiplied by the MET value to calculate the total METs. A database was created to record the PA codes (obtained from the compendium) used for each activity to ensure consistency among the participants' activities. For example, all moderate-intensity walking was coded as: 17,220, walking, $4.0 \mathrm{~m} / \mathrm{h}$ level, firm surface, very brisk pace, with an MET value of 5.0. For activities that could be classified as several different MET items or if a MET code did not exist for an activity, consensus through discussion was reached by 2 authors (T.L. and J.R.H.) to determine the best fit for the particular activity. For example, an MET code did not exist for the activity pickleball. This was a recurring activity among participants, and therefore consensus was reached to use code 15,500, with an MET value of 6.0 (paddleball, casual, general). In addition, the proportion of participants meeting the genetic (personalized) and population-based PA targets was assessed in 
Table 1. Genetic variation among the participants in the GLB + LGx group

\begin{tabular}{|c|c|c|c|}
\hline PA component, gene (SNP) & $\begin{array}{l}\text { Genotype } \\
\text { distribution } \\
(n, \%)\end{array}$ & $\begin{array}{l}\text { Participants with an enhanced } \\
\text { response/athletic advantage genotype } \\
(n, \%)\end{array}$ & $\begin{array}{l}\text { Associated genetic-based } \\
\text { information }\end{array}$ \\
\hline Endurance, NRF2 (rs12594956) & $\begin{array}{l}\text { AA }(22,31.4) \\
\text { AC }(34,48.6) \\
\text { CC }(14,20.0)\end{array}$ & \multirow{2}{*}{$\begin{array}{l}\text { Enhanced athletic advantage }(14,20.0) \text {; } \\
\text { ultra-athletic advantage }(2,2.9)\end{array}$} & \multirow{2}{*}{$\begin{array}{l}\text { Genetic predisposition to excel in } \\
\text { endurance-based PA (based on an } \\
\text { algorithm of } 4 \text { genotypes) }\end{array}$} \\
\hline Endurance, NFIA-AS2 (rs1572312) & $\begin{array}{l}\text { AA }(1,1.4) \\
\text { CA }(8,11.4) \\
\text { CC }(61,87.1)\end{array}$ & & \\
\hline Strength/Power, ACTN3 (rs1815739) & $\begin{array}{l}\text { TT }(21,30.0) \\
\text { TC }(30,42.9) \\
\text { CC }(19,27.1)\end{array}$ & $\begin{array}{l}\text { Enhanced athletic advantage }(30,42.9) \text {; } \\
\text { ultra-athletic advantage }(19,27.1)\end{array}$ & $\begin{array}{l}\text { Genetic predisposition to excel in } \\
\text { strength-based PA }\end{array}$ \\
\hline
\end{tabular}

order to measure PA adherence. Furthermore, differences between groups in total minutes of moderate- and high-intensity activity over time were assessed. Adherence to PA targets was dependent on group and genotype (for the GLB + LGx group). For the standard GLB group and the GLB + LGx "typical weight loss response" group (FTO rs9939609 TA or TT genotype), participants were considered to be adhering to the PA guidelines if they completed at least $150 \mathrm{~min} /$ week of moderate- and/or high-intensity activity. For the GLB + LGx "enhanced weight loss response" group (FTO rs9939609 AA genotype), adherence was considered to be at least $30-60 \mathrm{~min} /$ day, 6 days a week ( $\geq 180-360 \mathrm{~min} /$ week) of moderateand/or high-intensity activity.

SPSS version 26.0 was used to conduct all statistical analyses. Extreme (far out) outliers, defined as \pm 3 IQR, were identified using SPSS Descriptive Statistics and removed (e.g., 12,150 weekly leisure time METs were calculated for one participant who reported 7.5 consecutive hours per day of leisure time activity, 6 days a week), as these were likely a reflection of over-reporting (online suppl. material, for all online suppl. material see www.karger.com/ doi/10.1159/000510216). Split plot analysis of covariance was used to analyze between-group differences in the amount ( $\mathrm{min}$ ) of moderate- and high-intensity weekly leisure time PA, as well as the total weekly leisure time METs from baseline to 3, 6, and 12 months of follow-up. Binary logistic regression (generalized linear models) was used to assess differences between groups for the proportion of participants adhering to the PA guidelines at each time point. Baseline PA levels ( $\mathrm{min}, \mathrm{MET}$, and adherence) were controlled for in all of the analyses, with $p<0.05$ considered statistically significant.

\section{Results}

Table 1 indicates the genetic results of the participants randomized to the GLB + LGx group. Weekly MET were significantly higher in the GLB + LGx group compared to the standard GLB group at 6 months (Table 2). For weekly minutes of PA, while not statistically significant $(p=$ 0.18 ; Table 2), only the GLB + LGx group achieved $>150$ $\mathrm{min} /$ week of moderate- and high-intensity PA, on average. There were no significant differences in adherence to the PA targets in the GLB + LGx group compared to the standard GLB group ( $p=0.25$ at 3 months, $p=0.18$ at 6 months, and $p=0.99$ at 12 months). Overall, the GLB + LGx intervention led to an enhanced leisure time PA energy expenditure after the 6-month follow-up, compared to the standard, population-based GLB intervention.

\section{Discussion}

Our findings demonstrated that the provision of genetic-based PA information and advice enhanced leisure time PA energy expenditure after the moderate-term, 6-month 
Table 2. Weekly duration of moderate + high-intensity PA and weekly MET

\begin{tabular}{llrlll}
\hline & Baseline & \multicolumn{1}{l}{3 months } & 6 months & 12 months \\
\hline PA, min/week & & & & \\
GLB & $90.1 \pm 0.0(90.1-90.1)$ & $122.8 \pm 23.7$ & $75.4-170.2)$ & $114.8 \pm 26.3(62.2-167.3)$ & $144.6 \pm 24.6(95.4-193.7)$ \\
GLB + LGx & $90.1 \pm 0.0(90.1-90.1)$ & $136.2 .0 \pm 23.4$ & $89.5-182.8)$ & $169.2 \pm 25.9(117.4-221.0)$ & $112.8 \pm 24.364 .4-161.2)$ \\
\hline MET, n/week & & & & & \\
GLB & $727.9 \pm 0.0(727.9-727.9)$ & $1,071.0 \pm 142.0$ & $787.6-1,354.3)$ & $\mathbf{0 . 6 2 1 . 6} \pm \mathbf{1 4 1 . 9 ( 3 3 8 . 4 - 9 0 4 . 8 )}$ & $951.5 \pm 126.9(698.3-1,204.7)$ \\
GLB + LGx & $727.9 \pm 0.0(727.9-727.9)$ & $884.3 \pm 142.0$ & $600.9-1,167.6)$ & $\mathbf{1 , 1 1 4 . 7 \pm 1 4 1 . 9 ( 8 , 3 1 . 5 - 1 , 3 9 7 . 8 )}$ & $818.5 \pm 126.9(565.2-1,071.7)$ \\
\hline
\end{tabular}

Values are presented as means $\pm \mathrm{SE}(95 \% \mathrm{CI})$. Values in bold are statistically significant. $p=0.01 ; n=71$ (GLB, $n=35 ; \mathrm{GLB}+\mathrm{LGx}$, $n=36$ ).

follow-up. This demonstrates that genetically tailored PA information and advice exhibit clinical potential. Future research should explore how this enhanced leisure time PA energy expenditure can be sustained in the long term given that leisure time PA energy expenditure contributes to improved cardiometabolic, brain, and bone health among others [29]. Some prior studies have also shown that disclosure of genetic information can enhance PA habits [30, 31 ], though research in this area has been inconsistent [3234]. Furthermore, much of the research in this area has provided participants with genetic interventions that were unlikely to facilitate changes in PA habits based on established theory and knowledge on facilitating health behavior changes $[21,26]$. For example, in one study measuring PA outcomes resulting from disclosure of genetic susceptibility to Alzheimer disease, if participants inquired about any prevention strategies for the onset of Alzheimer disease, the presenter would communicate that there "are no effective methods of treatment or prevention to date" [34]. Thus, it is perhaps not surprising that this study found no change in PA habits [34]. Therefore, genetic interventions need to consider current knowledge on how to best facilitate behavior changes if they are aiming to facilitate successful lifestyle change outcomes.

While the present study demonstrated greater leisure time PA METs in the GLB + LGx group after the moderateterm, 6-month follow-up, this greater engagement in PA with the provision of genetic information and advice was not enhanced in the short term (3 months) or the long term (12 months). Given that the GLB and GLB + LGx interventions were highly intensive, with 23 group-based sessions, and focused on several lifestyle changes (predominantly nutrition and PA), it is possible that participants were focused on other lifestyle components of the interventions during the first 3 months and/or the last 6 months. In addition, the final 6 months of the GLB curriculum include a minimal focus on moderate- to high-intensity PA, with only 2 PA-related sessions, primarily focused on sitting less, good posture, and stretching [23]. This was a limitation of the intervention. We hypothesize that a greater focus on moderate-to-high intensity PA during the second half of the interventions (for both intervention groups) would have resulted in the 6-month findings being sustained in the long term; future research should seek to explore this. In addition, the sample size may have limited our abilities to detect significant differences in the exploratory analyses. Moreover, given the study population, the results are primarily generalizable to middle-aged, Caucasian women with obesity who are interested in weight management.

Nonetheless, our results indicate that it is possible to use genetic information and advice to motivate individuals to enhance PA energy expenditure. The NOW trial communicated the PA information and recommendations within the GLB program, which is a gold-standard, group-based, lifestyle change weight management program but is only offered in a select few clinics in Canada and approximately 50 healthcare facilities in the USA [35]. Therefore, future pragmatic research should further seek to assess PA outcomes resulting from a genetic intervention compared to the standard of care, which typically consists of the communication of population-based $\mathrm{PA}$ recommendations in a one-on-one setting with a healthcare provider. It should be noted that standard of care typically requires a patient to purchase an LGx test, which is not feasible for all at a public health level. In addition, future research should aim to assess other outcomes resulting from LGx interventions such as measures of cardiometabolic health, stress, the types of PA that participants engaged in, and strength/endurance level. This future work should also use direct measures of PA, such as through the use of accelerometers, rather than self-reported data. 


\section{Conclusion}

This is the first study to assess changes in PA resulting from a population-based versus a genetic-based intervention within a weight management program. Overall, results from the NOW trial provide some promise to the body of research evaluating the impact of genetic-based recommendations on PA behaviors. While the provision of an LGx intervention resulted in a greater leisure time PA energy expenditure after a 6-month follow-up, future research should determine how this could be sustained over the long term.

\section{Statement of Ethics}

This study was approved by the Western University Research Ethics Board (108511) and all of the participants provided written informed consent.

\section{Conflict of Interest Statement}

The authors have no conflicts of interest to declare.

\section{Funding Sources}

This project was supported by a grant from Brescia University College. Nutrigenomix Inc. provided complimentary genetic test kits and laboratory analyses. While J.R.H. has formerly completed work for Nutrigenomix Inc. as a professional consulting dietitian, she has not consulted for the company since January 2017. She received no payment or direction to conduct this study or prepare this article from Nutrigenomix Inc. The article resulted from her independent doctoral studies at Western University.

J.R.H. was supported through an Ontario Graduate Scholarship, an Ontario Respiratory Care Society Fellowship, and a Canadian Institutes of Health Research Frederick Banting and Charles Best Doctoral Award.

\section{Author Contributions}

J.R.H., J.G., J.A.S, C.O., and J.M. were involved in designing this study. J.R.H. conducted this study at the East Elgin Family Health Team. T.L. calculated METs, with supervision from J.R.H. T.L., J.R.H. and J.A.S. completed the statistical analyses. T.L. and J.R.H. wrote the first draft of this paper and revised subsequent drafts. All of the authors revised the drafts, approved the final version of this paper, and are accountable for all aspects of this work.

\section{References}

1 Penedo FJ, Dahn JR. Exercise and well-being: a review of mental and physical health benefits associated with physical activity. Curr Opin Psychiatry. 2005 Mar;18(2):189-93.

2 Ozemek C, Lavie CJ, Rognmo Ø. Global physical activity levels - Need for intervention [Internet]. Prog Cardiovasc Dis. 2019 Mar - Apr; 62(2):102-7.

3 Frayling TM, Timpson NJ, Weedon MN, Freathy RM, Lindgren CM, Perry JRB, et al. A common variant in the FTO gene is associated with body mass index and predisposes to childhood and adult obesity. Science. 2007 May;316(5826):889-94.

4 Sonestedt E, Gullberg B, Ericson U, Wirfält E, Hedblad B, Orho-Melander M. Association between fat intake, physical activity and mortality depending on genetic variation in FTO. Int J Obes. 2011 Aug;35(8):1041-9.

5 Yang Q, Xiao T, Guo J, Su Z. Complex relationship between obesity and the fat mass and obesity locus. Int J Biol Sci. 2017 May 15; 13(5):615-29.

6 Celis-Morales C, Marsaux CF, Livingstone KM, Navas-Carretero S, San-Cristobal R, O'donovan CB, et al.; Food4Me Study. Physical activity attenuates the effect of the FTO genotype on obesity traits in European adults: The Food4Me study. Obesity (Silver Spring). 2016 Apr;24(4):962-9.
7 Andreasen $\mathrm{CH}$, Stender-Petersen KL, Mogensen MS, Torekov SS, Wegner L, Andersen $\mathrm{G}$, et al. Low physical activity accentuates the effect of the FTO rs9939609 polymorphism on body fat accumulation. Diabetes. 2008 Jan; 57(1):95-101.

8 Zou ZC, -J Mao L, Shi YY, Chen JH, Wang LS, Cai W. Effect of exercise combined with dietary intervention on obese children and adolescents associated with the FTO rs9939609 polymorphism [Internet]. Eur Rev Med Pharmacol Sci. 2015 Dec;19(23):4569-75.

9 Speakman JR. The 'Fat Mass and Obesity Related' (FTO) gene: Mechanisms of Impact on Obesity and Energy Balance. Curr Obes Rep. 2015 Mar;4(1):73-91.

10 Kilpeläinen TO, Qi L, Brage S, Sharp SJ, Sonestedt E, Demerath E, et al. Physical activity attenuates the influence of FTO variants on obesity risk: a meta-analysis of 218,166 adults and 19,268 children. PLoS Med. 2011 Nov; 8(11):e1001116.

11 Zhu J, Loos RJ, Lu L, Zong G, Gan W, Ye X, et al. Associations of genetic risk score with obesity and related traits and the modifying effect of physical activity in a Chinese Han population. PLoS One. 2014 Mar;9(3):e91442.

12 Santiago C, Ruiz JR, Buxens A, Artieda M, Arteta D, González-Freire M, et al. Trp64Arg polymorphism in ADRB3 gene is associated with elite endurance performance. Br J Sports Med. 2011 Feb;45(2):147-9.
13 He Z, Hu Y, Feng L, Lu Y, Liu G, Xi Y, et al NRF2 genotype improves endurance capacity in response to training. Int J Sports Med. 2007 Sep;28(9):717-21.

14 Zarebska A, Jastrzebski Z, Kaczmarczyk M, Ficek K, Maciejewska-Karlowska A, Sawczuk $\mathrm{M}$, et al. The GSTP1 c.313A $>\mathrm{G}$ Polymorphism Modulates The Cardiorespiratory Response to Aerobic Training. Biol Sport. 2014 Dec;31(4):261-6.

15 Ahmetov I, Kulemin N, Popov D, Naumov V, Akimov E, Bravy Y, et al. Genome-wide association study identifies three novel genetic markers associated with elite endurance performance. Biol Sport. 2015 Mar;32(1):3-9.

16 Kikuchi N, Miyamoto-Mikami E, Murakami $\mathrm{H}$, Nakamura T, Min SK, Mizuno M, et al. ACTN3 R577X genotype and athletic performance in a large cohort of Japanese athletes. Eur J Sport Sci. 2016 Sep;16(6):694-701.

17 Yang N, MacArthur DG, Gulbin JP, Hahn AG, Beggs AH, Easteal S, et al. ACTN3 genotype is associated with human elite athletic performance [Internet]. Am J Hum Genet. 2003 Sep;73(3):627-31.

18 Ma F, Yang Y, Li X, Zhou F, Gao C, Li M, et al. The association of sport performance with ACE and ACTN3 genetic polymorphisms: a systematic review and meta-analysis. PLoS One. 2013;8(1):e54685. 
19 Eynon N, Duarte JA, Oliveira J, Sagiv M, Yamin C, Meckel Y, et al. ACTN3 R577X polymorphism and Israeli top-level athletes. Int J Sports Med. 2009 Sep;30(9):695-8.

20 Mutch DM, Zulyniak MA, Rudkowska I, Tejero ME. Lifestyle Genomics: Addressing the Multifactorial Nature of Personalized Health. Lifestyle Genomics. 2018;11(1):1-8.

21 Horne J, Madill J, O’Connor C, Shelley J, Gilliland J. A Systematic Review of Genetic Testing and Lifestyle Behaviour Change: Are We Using High-Quality Genetic Interventions and Considering Behaviour Change Theory? Lifestyle Genomics. 2018;11(1):49-63.

22 Horne J, Madill J, Gilliland J. Incorporating the 'Theory of Planned Behavior' into personalized healthcare behavior change research: a call to action [Internet]. Per Med. 2017 Nov; 14(6):521-9.

23 Horne J, Gilliland J, O'Connor C, Seabrook J, Hannaberg P, Madill J. Study protocol of a pragmatic randomized controlled trial incorporated into the Group Lifestyle Balance ${ }^{\mathrm{TM}}$ program: the nutrigenomics, overweight/ obesity and weight management trial (the NOW trial). BMC Public Health. 2019;19(1): 310.

24 Horne J, Gilliland J, O’Connor C, Seabrook J, Madill J. Change in weight, BMI and body composition in a population-based intervention versus genetic-based intervention: The NOW Trial. Obesity. 2020;28(8):1419-27.
25 Horne J, Gilliland J, O’Connor C, Seabrook J, Madill J. Enhanced long-term dietary change and adherence in a nutrigenomics-guided intervention compared to a population-based (GLB/DPP) lifestyle intervention for weight management: Results from the NOW randomized controlled trial. BMJ Nutr Prev Heal. 2020;3(1).

26 Ajzen I. The theory of planned behavior. Organizational Behav Hum Decis Process. 1991; 50(2):179-211.

27 Holtermann A, Krause N, van der Beek AJ, Straker L. The physical activity paradox: six reasons why occupational physical activity (OPA) does not confer the cardiovascular health benefits that leisure time physical activity does. Br J Sports Med. 2018 Feb;52(3): 149-50.

28 Ainsworth BE, Haskell WL, Herrmann SD, Meckes N, Bassett DR Jr, Tudor-Locke C, et al. 2011 Compendium of Physical Activities: a second update of codes and MET values. Med Sci Sports Exerc. 2011 Aug;43(8):157581.

29 Department of Health \& Human Services. Physical Activity Guidelines Advisory Committee. Phys Act Guidel Advis Comm Sci Rep [Internet]. 2018;2018:779.
30 Egglestone C, Morris A, O’Brien A. Effect of direct-to-consumer genetic tests on health behaviour and anxiety: a survey of consumers and potential consumers. J Genet Couns. 2013 Oct;22(5):565-75.

31 Kaufman DJ, Bollinger JM, Dvoskin RL, Scott JA. Risky business: risk perception and the use of medical services among customers of DTC personal genetic testing. J Genet Couns. 2012 Jun;21(3):413-22.

32 Meisel SF, Beeken RJ, van Jaarsveld CH, Wardle J. Genetic susceptibility testing and readiness to control weight: results from a randomized controlled trial. Obesity (Silver Spring). 2015 Feb;23(2):305-12.

33 Hietaranta-Luoma HL, Tahvonen R, Iso-Touru T, Puolijoki H, Hopia A. An intervention study of individual, apoE genotype-based dietary and physical-activity advice: impact on health behavior. J Nutrigenet Nutrigenomics. 2014;7(3):161-74.

34 Vernarelli JA, Roberts JS, Hiraki S, Chen CA, Cupples LA, Green RC. Effect of Alzheimer disease genetic risk disclosure on dietary supplement use. Am J Clin Nutr. 2010 May;91(5): 1402-7.

35 University of Pittsburgh. GLB program locations [Internet]. Available from: https://www. diabetesprevention.pitt.edu/index.php/forthe-public/glb-program-locations-unitedstates/. 